\title{
Etiological Profile of 11 Cases of Bud-Chiari Syndrome
}

\author{
Salim Larbani*, Nabila Lyazidi, Mohamed Hamza Bouchouareb, Amir \\ Hadji, Yasmine Hamma, Chafia Ouarab, Sadek Boumedine and Soraya \\ Ayoub \\ Internal Medicine Department, University Hospital Centre Issad Hassani, Beni Messous \\ Algiers, Algeria \\ *Corresponding Author: Salim Larbani, Internal Medicine Department, University \\ Hospital Centre Issad Hassani, Beni Messous Algiers, Algeria.
}

Received: March 18, 2020

Published: April 07, 2020

(c) All rights are reserved by Salim Larbani., et al.

\begin{abstract}
Background and Aim: Budd-Chiari syndrome is a relatively rare condition with multiple etiologies and high risk of complications and death. The aim of our study were to investigate the etiology of BCS, especially auto-inflammatory diseases and rare associations, analyze different clinical presentations, treatment modalities and evolutionary profile.

Methods: Retrospective cohort study of patients hospitalized from 2000 to 2018 with confirmed diagnosis of BCS on Doppler ultrasound, with which we have conducted a large etiological survey.

Results: 11 patients were included; mean age 29 years (16 to 45 years); sex ratio (M/F) 0,83.

All patients were symptomatic at diagnosis with classic subacute to chronic clinic presentation, 6 patients had extravascular manifestations.

The etiological survey conclusion was: Behçet's syndrome in 6 cases (most severe forms), liver chronic disease in 2 cases (hepatitis C and cryptogenic cirrhosis), protein S deficiency associated with coeliac disease in 1 case, paroxysmal nocturnal hemoglobinuria associated with antiphospholipid syndrome in 1 case and factor five Leiden mutation in 1 case. The treatment included anticoagulants in 7 cases, specific treatment combining corticosteroids and immunosuppressive drugs in patients with BS, Gluten-free diet in celiac disease.

Conclusion: Behçet's syndrome was the first etiology in our study, it must be systematically searched in countries with high prevalence, especially in young patients with extravascular manifestations. The association of 2 or plus prothrombotic states is not rare and often insidious. The systematic search of this kind of association must be discussed.

Keywords: Budd-Chiari Syndrome; Behçet's Syndrome; Portal Hypertension; Vasculitis; Thrombosis
\end{abstract}

\section{Abbreviations}

AAE: Anti-Endomysium; AATG: Anti-Transglutaminase; ANA: Antinuclear Antibody; ANCA: Antineutrophilic Cytoplasmic Antibody; APS: Antiphospholipid Antibody Syndrome; BCS: Budd-Chiari Syndrome; BS: Behçet's Syndrome; CD: Coeliac Disease; CBC: Complete Blood Count; CT: Computed Tomography; DVT: Deep Venous Thrombosis; FVLM: Factor Five Leiden Mutation; HV: Hepatic Vein; ICBD: International Criteria for Behçet's Disease; IVC: Inferior Vena Cava; MRI: Magnetic Resonance Imaging; PHT: Portal Hypertension; PNH: Paroxysmal Nocturnal Hemoglobinuria; PV: Portal Vein; RA: Right Atrium; SVT: Superficial Venous Thrombosis

\section{Introduction}

Budd-Chiari syndrome (BCS) includes all clinical manifestations related to an obstruction of the hepatic venous outflow tract and/or supra-hepatic portion of the inferior vena cava (IVC), in the absence of sinusoidal obstruction syndrome, right heart failure or constrictive pericarditis [1].

BCS is a rare disease whose prevalence and incidence vary worldwide: more frequent in Asia, with incidences of $0.13 / \mathrm{M}$ in- habitants/year in Japan, 4.5 in China and 2.5 in Nepal vs 0.5 in Denmark and 0.9 in France; and prevalence of 2.4 pmh/year in Japan, 64 in China vs 1.4 in Sweden and 4.02 in France [2,3]. Overall, its prevalence is estimated approximately at 1/100,000 in the world population [4].

BCS is classified as primary or secondary according to the etiology.

Secondary forms are linked to a compression effect (mainly pyogenic infectious processes, amoebae, hydatidosis, echinococcosis, but also benign or, more rarely, malignant tumors, hematomas, etc.) or vascular invasion by tumors [5,6].

Primary forms are dominated by prothrombotic and hypercoagulation states ( $60 \%$ of patients in Western countries), which can be either hereditary/primary (factor V Leiden $[7,8]$, protein C, S or antithrombin III deficiency...) [9] or acquired/secondary (myeloproliferative disorders, paroxysmal nocturnal hemoglobinuria (PNH) [10] or antiphospholipid syndrome (APS) [7]). 
Autoimmune and auto-inflammatory diseases (Behçet's syndrome (BS) [11,12], celiac disease (CD), immunoallergic vasculitis, granulomatosis such as sarcoidosis...) are rare causes of BCS. These are potentially serious and dangerous pathologies requiring an early diagnosis for a specific adapted treatment. Anticoagulant treatment is often at the forefront associated with symptomatic and especially etiological treatment. The prognosis depends on the etiology, the earliness of the diagnosis and the speed with which treatment is started.

\section{Aim of the Study}

The aim of our study were to investigate the etiology of BCS, especially auto-inflammatory diseases and rare associations, analyze different clinical presentations, treatment modalities and evolutionary profile.

\section{Materials and Methods}

This retrospective study enrolled 11 cases of patients with BCS between 2000 and 2018. These patients were hospitalized in the Internal Medicine department for various reasons (exploration of chronic abdominal pain, disturbances of the liver balance, ascites...). Patients were confirmed to have BCS by non-invasive imaging methods: Doppler ultrasound and CT scan. The diagnosis criteria were the demonstration of direct or indirect signs obstruction of the hepatic veins (HV) and/or the IVC and hepatic venous collaterals.

All patients were hospitalized and have benefited from a full clinical and a large etiological investigation including:

- Broad biological assessment for all patients for diagnostic and prognostic purposes:

- Renal function and liver profile, a complete blood count (CBC) and inflammatory assessment for all patients.

- Hepatitis B and C virological markers were searched for systematically.

- Coagulation profile and thrombophilia assessment: homocysteine; anti-cardiolipin antibodies and anti-beta 2 glycoprotein antibodies; paroxysmal nocturnal hemoglobinuria (PNH), factor V Leiden mutation (FVLM); activated protein $\mathrm{C}$ resistance (APCR); coagulation protein deficiencies (C, S, anti-thrombin III).

- Systematic search for organ specific autoantibodies (antismooth muscle, anti-LKM and anti-mitochondrial antibodies; coeliac serology (anti-transglutaminase (AATG) anti-endomysium (AAE)) and non-organ specific antibodies (ANA and ANCA).

- We also looked for the diagnosis of myeloproliferative disorder in 4/10 patients with Bone-marrow biopsy and search for JAK2 mutation.

- Three exams were systematically applied on our patients:

- Doppler ultrasound to confirm the diagnosis of BCS and make a cartography of the vessels affected, look for portal hypertension PHT, specify liver and splenic size, status of ascites and search of lymph nodes or deep tumors. Heart involvement and right atrial thrombus were systematically searched by Doppler echocardiography.

- CT scan systematically performed in all patients to complete the mapping of affected vessels (vein or arteries) and also specify the characteristics of any associated visceral lesions.

- Upper endoscopy to look for signs of PHT (portal hypertensive gastropathy, esophageal varices) or other signs of other etiologies such as coeliac disease.

Results

Demographic characteristics

The present study enrolled 11 patients. They all had a primary BCS. Slight dominance of female gender with a sex ratio of 0,83 (5 men and 6 women). The mean age of patients was 29 years, ranging from 16 to 45 years.

\section{Clinical and paraclinical data}

All patients were symptomatic at diagnosis (Table 1 summarizes the clinical signs). The clinical course was from subacute to chronic in all cases. All patients presented a deterioration in the general condition and painful hepatomegaly, accompanied by an inaugural ascitic decompensation in 8 patients and PHT in 7 patients.

\begin{tabular}{|l|c|}
\hline \multicolumn{1}{|c|}{ Clinical characteristics } & Number \\
\hline Deterioration in the general condition & 11 \\
\hline Fever & 3 \\
\hline Painful hepatomegaly & 11 \\
\hline Splenomegaly & 6 \\
\hline Icterus & 6 \\
\hline Extra-intestinal manifestations & 5 \\
\hline Oral ulcers & 2 \\
\hline Genital ulcers & 4 \\
\hline Papulopustular lesions & 0 \\
\hline Erythema nodosum & 0 \\
\hline Skin pathergy reaction & 4 \\
\hline Uveitis & 6 \\
\hline Arthralgies/Arthrites & 0 \\
\hline Neurological manifestations & 0 \\
\hline Deep venous thrombosis & 1 \\
\hline Superficial venous thrombosis & 1 \\
\hline Pulmonary embolism & 0 \\
\hline Pleurisy & 0 \\
\hline Heart failure & 0 \\
\hline Pericarditis & 1 \\
\hline Large vessel arterial thrombosis & 1 \\
\hline Aneurism & \\
\hline
\end{tabular}

Table 1: Clinical characteristics of BS patients.

2 patients had a positive family history of BS. 6 patients' extraintestinal symptoms: oral ulcers in 5 cases, papulopustular lesions in 4 cases, genital ulcers in 2 patients, ophthalmological involvement in 4 cases: 1 case of anterior uveitis and 3 cases of severe panuveitis with a significant decrease in visual acuity. 
All patients had at least one cytopenia, (6/11 thrombocytopenia), liver function disturbances with (Cytolysis and/or cholestasis) with inflammatory syndrome.

Other biological exams: thrombophilia disorders in 2 cases coeliac serology positive in 1 case, $C$ viral serological in 1 case, antiphospholipid antibodies in 1 case, flow cytometry quantitating CD55 and CD59 positive in 1 case.

Upper endoscopy shown portal hypertensive gastropathy with esophageal varices in 6 cases (4 patients Grade 3 esophageal varices and red signs) and 1 patient had an aspect of duodenal atrophy which was biopsied.

\section{Vascular assessment}

All patients presented thrombosis of at least 2 of the 3 hepatic veins HVs (diagnosis criteria).

4 patients had isolated HV thrombosis, the others had either an extension of the thrombosis or another vascular localization:

- IVC thrombosis in 4 cases with extension to the right atrium (RA) in 3 cases.

- Portal vein (PV) thrombosis in 2 cases.

2 patients had a positive family history of deep vein thrombosis (DVT) and 1 patient had a history of superficial venous thrombosis (SVT).

2 patients had also an arterial involvement: aortic thrombosis in 1 case and aneurysm in the other.

\section{Etiologies}

Table 2 summarizes the different etiologies and the corresponding thrombosis sites.

\begin{tabular}{|l|c|c|c|c|}
\hline Patients & Age & Sex & Thrombosis site & Etiology \\
\hline 1 & 38 & F & $\begin{array}{c}3 \text { HV + IVC + RA } \\
+ \text { PV }\end{array}$ & Behçet's syndrome \\
\hline 2 & 27 & F & 3 HV + IVC + RA & Behçet's syndrome \\
\hline 3 & 32 & M & 3 HV + IVS + RA & Behçet's syndrome \\
\hline 4 & 32 & F & 3 HV + IVC + RA & Behçet's syndrome \\
\hline 5 & 24 & M & $3 \mathrm{HV}+$ PV + & Behçet's syndrome \\
\hline 6 & 16 & F & $3 \mathrm{HV}+$ SVT & Behçet's syndrome \\
\hline 7 & 22 & M & $2 \mathrm{HV}+$ DVT & PNH + APS \\
\hline 8 & 41 & M & $2 \mathrm{HV}$ & Hepatitis C cirrhosis \\
\hline 9 & 45 & M & $2 \mathrm{HV}$ & Cryptogenic cirrhosis \\
\hline 10 & 18 & F & $2 \mathrm{HV}$ & $\begin{array}{c}\text { Facteur Five Leiden } \\
\text { mutation }\end{array}$ \\
\hline 11 & 24 & F & $2 \mathrm{HV}$ & $\begin{array}{c}\text { Protéine S deficiency + } \\
\text { Coeliac disease }\end{array}$ \\
\hline
\end{tabular}

Table 2: Etiologies of BCS and thrombosis sites.

APS: Antiphospholipid Syndrome; DVT: Deep Vein Thrombosis; HV: Hepatic Vein ; IVC: Inferior Vena Cave; RA: Right Atrium; PNH:

Paroxysmal Nocturnal Hemoglobinuria; PV: Portal Vein; SVT: Superficial Venous Thrombosis.
More than half of the patients (6/10) presented with Behçet BS disease. The diagnostic was defined according to the International Criteria for BS (ICBD) [13]. The patient was classified as having BS with 4 or more points.

2 Patients had liver chronic disease: hepatitis C cirrhosis in 1 case and cryptogenic cirrhosis in the other.

2 Patients had thrombophilia disorder: 1 case of factor $V$ Leiden mutation and 1 case of protein $\mathrm{S}$ deficiency associated with coeliac disease.

1 patient had PNH associated with antiphospholipid syndrome (APS).

\section{Treatment}

7 patients were treated by pharmacological anticoagulation (low molecular weight heparin, $100 \mathrm{unit} / \mathrm{kg} / 12 \mathrm{~h}$ followed by acenocoumarol) including 2 cases of BS, the other patients had significant hemostasis disorders (4 cases of BS).

The 6 patients with BS presented severe forms which required corticosteroids (3 boluses intravenous methylprednisolone of 0.5 $1 \mathrm{~g} /$ day, followed by oral prednisone $0.5-1 \mathrm{mg} / \mathrm{kg} / \mathrm{d}$ ) associated to an immunosuppressive therapy (cyclophosphamide $600 \mathrm{mg}$, only 1 patient with stable clinical state and pregnancy desire, received azathioprine $2 \mathrm{mg} / \mathrm{kg} / \mathrm{d}$ ).

Gluten-free diet for the patient with celiac disease.

\section{Evolutionary profile}

- Clinical outcome was favorable with restoration of venous outflow for the patients with thrombophilia disorder and PNH. Unfavorable with progressive worsening and refractory ascites: for the 2 patients with liver cirrhosis.

- Death of 3 patients with BS by hemorrhagic complications after an average evolution of 6 months, the 3 others currently remain clinically stable.

\section{Discussion}

Our study confirmed relatively young age in BCS, especially in patients with thrombophilia disorders and BS as reported in Bayraktar., et al. study [12]. The clinical course in BCS is generally chronic as showed in old Japanese study [14], however this was not the case in our study, especially for patients with BS who had mainly a subacute presentation, its prognosis was determined by the severity of this disease. We noticed some characteristics of BCS due to BS as oral ulcers, genital ulcers, arthritis, papulopustular lesions and uveitis which have been noted in several studies as Desbois., et al. study [15].

Despite the stereotypical nature of the initial BCS picture, evolutionary differences were noted, probably in relation to the etiology on the one hand, the severity and extent of the thromboses on the other. 
The anticoagulant treatment is the cornerstone of the treatment; however, it must be adapted to the patient's profile and the balance of hemorrhagic risk/thrombotic risk. In our series, 4 patients did not receive anticoagulant therapy for significant hemostasis disorders.

The etiological profile was clearly dominated by BS 6/11 and the affected patients presented the most severe forms, these results are higher than the average of other studies, indeed BS is an uncommon cause of BCS and its prevalence in the Mediterranean, Central Asia, and Far East countries is significantly higher than in Europe and the US [16,17].

BS was reported in 5\%, 9\% and 14,4\% of BCS 'patients in western countries, Turkey [18] and Egypt respectively (cohort of 271 [19]).

BCS is the most severe venous manifestation of BS and is associated with a 9-fold increase in mortality in these patients [20]. The prevalence of BCS among patients with BS is variable in the literature (0.3 - 3.2\%) [21,22]. In a turkish cohort (Seyahi., et al.) of 9000 patients with BS, 43 had BCS as a complication.

BCS is associated with a high risk of complications and death due to PHT and liver failure (6/10 of our patients had PHT).

The thrombotic process during bs is probably multifactorial, associating an inflammatory state responsible of hypercoagulability with endothelial lesions of vasculitis on, probably, a predisposed genetic background.

The specific treatment of BCS in BS associate, systematically and immediately, corticosteroids and immunosuppressants. Anticoagulation therapy is debated in this indication due to the etiopathogenesis of vascular injury and thrombosis arising from an intense inflammatory process [23,24]. IVC thrombosis can be an exception to the use of anticoagulants in patients with BS [25]. In our series, only $2 / 6$ of BS patients received anticoagulant therapy, the others presented a significant risk of hemorrhagic complications.

For the other etiologies: 2 patients had liver cirrhosis (hepatitis $\mathrm{C} /$ cryptogenic), it should be noted that all inflammatory processes in the liver can be complicated by portal thrombosis and more rarely by BCS [26]. 2 patients had hereditary hemostasis disorders and 2 others had acquired disorders.

A multicentric European study showed that the prothrombotic state is highlighted in $84 \%$ of the patients with primary BCS ( $\mathrm{n}=$ 163) and very often, several prothrombotic states are associated (46\% have at least 2 prothrombotic states) [27]. In our study, 2 patients presented rare associations culminating 2 prothrombotic states: (PNH + APS) and (Protein S deficiency + coeliac disease). This kind of insidious association must be searched, because spe- cific treatment may be necessary, that's why thrombophilia assessment must be systematically carried out in suspected cases. The diagnosis of celiac disease was suspected at upper endoscopy (aspect of duodenal atrophy) and confirmed by histological study and the serologies which were systematically performed in all the patients in our study.

No myeloproliferative syndrome was diagnosed, despite the fact that it can be implicated in about $50 \%$ of cases according to literature data [28].

Asymptomatic patients have a more favorable course with less than $10 \%$ of deaths [28]. All of our patients were symptomatic at the time of diagnosis, which partly explains the severity of the forms presented.

More than half of our BCS patients had BS and half of them died, showing the severity of this disease. This high mortality rate is similar to the results of other larger studies which can reach $47 \%$ (notably in a recent survey of 43 BCS) despite intensive treatment [29]. This shows the importance of early diagnosis for rapid specific management.

In the Egyptian cohort, the causes of death in patients with BS were liver cell failure and PHT in 4/9 patients and extensive pulmonary embolism in 2 patients and pulmonary metastasis from hepatocellular carcinoma in 1 patient.

In our study, the high mortality in patients with BS $(3 / 6)$ can be explained on the vascular tropism of BS responsible for the multiplicity and the extent of thromboses, indeed our patients had multiple vascular locations. Having a combined involvement of the 3 HV and IVC can be a poor prognostic indicator.

If the two left HV are obstructed, the left lobe can drain through the caudate lobe, which has its own venous system connected directly to the systemic circulation. The obstruction of the three HV does not allow this suppleness [30].

The thrombosis of IVC and the multiplicity of vascular locations in general, seem to be closely linked to the prognosis, particularly in BS [31]. In our study, all patients with BS had multiple vascular localizations (4/6 had IVC thrombosis with an extension to RA in 3 cases), which agree with the Egyptian cohort of Sakr., et al [19]. Indeed, there is. a significant association between BCS and IVC occlusion in patients with BS which can be combined with hepatic veins: $49 \%$, isolated: $2 \%$, is one of main causes of death [32].

Cardiac involvement, usually rare in BS (6\%), was found in 4/11 patients, non-negligible aggravating factors, responsible for a mortality rate. This complication generally occurs in young men from the Mediterranean basin and the Middle East and predominates in the right chambers of the heart [33]. 
Severe liver cell failure was also, a determining factor for poor prognosis which was at the origin of hemorrhagic complications contraindicating any anticoagulation therapy.

The limitations of this study include the relatively small number of patients with BCS (who were diagnosed and treated in an internal medicine department) its retrospective nature and the relatively small number of patients with BCS however, this study has shown that BS is one of the main etiologies of BCS and should be systematically searched, especially in young people with extravascular manifestations.

\section{Conclusion}

BCS is a relatively rare condition with multiple etiologies. Autoinflammatory diseases and thrombophilia represent a significant part of the etiological spectrum, particularly in young people.

BS is an uncommon etiology of BCS, but it was the first in our study. This can be explained by high prevalence of this disease in Mediterranean countries. It must be systematically searched in young patients with extravascular manifestations. The pathology is particularly severe and its potentially serious prognosis requires rapid and appropriate diagnosis and management. In addition to symptomatic treatment, the specific treatment must include corticosteroids and immunosuppressants as soon as possible.

The association of 2 or plus prothrombotic states is not rare and often insidious. The systematic search of this kind of association must be discussed as well.

\section{Bibliography}

1. Valla DC. "Primary Budd-Chiari syndrome”. Journal of Hepatology 50 (2009): 195-203.

2. Hadengue A., et al. "The changing scene of hepatic vein thrombosis: recognition of asymptomatic cases". Gastroenterology 106 (1994): 1042-1047.

3. Parker RGF. "Occlusion of the hepatic veins in man". Medicine (Baltimore) 38 (1959): 369-402.

4. Aydinli M and Bayraktar Y. "Budd-Chiari syndrome: etiology, pathogenesis and diagnosis". World Journal of Gastroenterology 13 (2007): 2693-2696.

5. Taylor RW., et al. "Leiomyosarcoma of the inferior vena cava presenting as Budd- Chiari syndrome”. Liver 7 (1987): 201205.

6. Schraut WH and Chilcote RR. "Metastatic Wilms' tumor causing acute hepatic-vein occlusion (Budd-Chiari syndrome) ". Gastroenterology 88 (1985): 576-579.

7. Janssen HL., et al. "Factor V Leiden mutation, prothrombin gene mutation, and deficiencies in coagulation inhibitors associated with Budd-Chiari syndrome and portal vein thrombosis: results of a casecontrol study". Blood 96 (2000): 23642368.
8. Denninger MH., et al. "Budd-Chiari syndrome and factor V Leiden mutation”. Lancet 345 (1995): 525-526.

9. Denninger MH., et al. "Cause of portal or hepatic venous thrombosis in adults: the role of multiple concurrent factors". Hepatology 31 (2000): 587-591.

10. Myones BL and McCurdy D. "The antiphospholipid syndrome: immunologic and clinical aspects. Clinical spectrum and treatment". The Journal of Rheumatology 27.58 (2000): 20-28.

11. Bismuth E., et al. "Hepatic vein thrombosis in Behcet's disease”. Hepatology 11 (1990): 969-974.

12. Bayraktar Y., et al. "Budd-Chiari syndrome: a common complication of Behcet's disease”. The American Journal of Gastroenterology 92 (1997): 858-862.

13. Yazici H., et al. "Behcet syndrome: is it one condition?" Clinical Reviews in Allergy and Immunology 43 (2012): 275-280.

14. Kuniyoshi Y., et al. "Surgical treatment of Budd-Chiari syndrome induced by Behcet's disease". Annals of Thoracic and Cardiovascular Surgery 8 (2002): 374-380.

15. Desbois AC., et al. "Immunosuppressants reduce venous thrombosis relapse in Behcet's disease". Arthritis and Rheumatology 64 (2012): 2753-2760.

16. Yurdakul S., et al. "The prevalence of Behcet's syndrome in a rural area in northern Turkey". The Journal of Rheumatology 15 (1988): 820-822.

17. Cakir N., et al. "Prevalence of Behcet's disease in rural western Turkey: a preliminary report". Clinical and Experimental Rheumatology 22 (2004): 53-55.

18. Aydinli M and Bayraktar Y. "Budd-Chiari syndrome: etiology, pathogenesis, and diagnosis". World Journal of Gastroenterology 13 (2007): 2683-2696.

19. Sakr MA., et al. "Characteristics and outcome of primary BuddChiari syndrome due to Behçet's syndrome". Clinics and Research in Hepatology and Gastroenterology (2019).

20. Calamia KT., et al. "Major vessel involvement in Behcet disease". Current Opinion in Rheumatology 17 (2005): 1-8.

21. Bayraktar Y., et al. "Budd-Chiari syndrome: a common complication of Behcet's disease". The American Journal of Gastroenterology 92 (1997): 858-862.

22. Korkmaz C., et al. "Budd-Chiari syn-drome in the course of Behcet's disease: clinical and laboratory analysis of four cases". Joint Bone Spine 74 (2007): 245-248.

23. Hatemi G., et al. "Update of the EULAR recommendations for the management of Behcet's syndrome". Annals of the Rheumatic Diseases 77 (2018): 808-818. 
24. Seyahi E. "Behcet's disease: how to diagnose and treat vascular involvement". Best Practice and Research: Clinical Rheumatology 30 (2016): 279-295.

25. Tayer-Shifman OE., et al. "Major vessel thrombosis in Behcet's disease: the dilemma of anticoagulant therapy e the approach of rheumatologists from different countries". Clinical and $E X^{-}$ perimental Rheumatology 30 (2012): 735-740.

26. Denninger MH., et al. "Cause of portal or hepatic venous thrombosis in adults: the role of multiple concurrent factors". Hepatology 31 (2000): 587-591.

27. Chait Y., et al. "Relevance of the criteria commonly used to diagnose myeloproliferative disorder in patients with splanchnic vein thrombosis". British Journal of Haematology 129 (2005): 553-560.

28. Deltenre P., et al. "Factor V Leiden related Budd-Chiari syndrome". Gut 48 (2001): 264-268.

29. Seyahi E., et al. "An outcome survey of 43 patients with BuddChiari syndrome due to Behcet's syndrome followed up at a single, dedicated center". Seminars in Arthritis and Rheumatism 44 (2015): 602-609.

30. Zeitoun G., et al. "Outcome of Budd-Chiari syndrome: a multivariate analysis of factors related to survival including surgical portosystemic shunting". Hepatology 30 (1990): 84-89.

31. Sakr MA., et al. "The pattern of vascular involvement in Egyptian patients with Budd-Chiari syndrome: relation to etiology and impact on clinical presentation". Annals of Hepatology 17 (2018): 638-644.

32. Darwish MS., et al. "Etiology, management, and outcome of the Budd-Chiari syndrome". Annals of Internal Medicine 151 (2009): 167-175.

33. Darie C., et al. "Pseudotumeur cardiaque révélant une maladie de Behçet”. Revue de Médecine Interne 26 (2005): 420-424.

\section{Assets from publication with us}

- Prompt Acknowledgement after receiving the article

- Thorough Double blinded peer review

- Rapid Publication

- Issue of Publication Certificate

- High visibility of your Published work

Website: https://www.actascientific.com/

Submit Article: https://www.actascientific.com/submission.php Email us: editor@actascientific.com

Contact us: +919182824667 